\title{
Integrated Management of Land Use Systems under Systemic Risks and Security Targets: A Stochastic Global Biosphere Management Model
}

\author{
Tatiana Ermolieva, Petr Havlík, Yuri Ermoliev, \\ Aline Mosnier, Michael Obersteiner, David Leclère, \\ Nikolay Khabarov, Hugo Valin and Wolf Reuter ${ }^{1}$
}

(Original submitted August 2014, revision received August 2015, accepted October 2015.)

\begin{abstract}
Interdependencies among land use systems resemble a complex network connected through demand-supply relationships. Disruption of this network may catalyse systemic risks affecting food, energy, water and environmental security (FEWES) worldwide. We describe the conceptual development, expansion and practical application of a stochastic version of the Global Biosphere Management Model (GLOBIOM), used to assess competition for land use between agriculture, bioenergy and forestry at regional and global scales. In the stochastic version of the model, systemic risks of various kinds are explicitly covered and can be analysed and mitigated in all their interactions. While traditional deterministic scenario analysis produces sets of scenario-dependent outcomes, stochastic GLOBIOM explicitly derives robust outcomes that leave the systems better-off, independently of which scenario applies. Stochastic GLOBIOM is formulated as a stochastic optimisation model that is critical for evaluating portfolios of robust interdependent decisions: ex-ante strategic decisions (production allocation, storage capacities) and ex-post adaptive (demand, trading, storage control) decisions. As an example, the model is applied to the question of optimal storage facilities, as buffers for production shortfalls, to meet regional and global FEWES requirements when extreme events occur. Expected shortfalls and storage capacities have a close relationship with Value-at-Risk (VaR) and Conditional Value-at-Risk (CVaR)
\end{abstract}

\footnotetext{
${ }^{1}$ All the authors are with the International Institute for Applied Systems Analysis, Ecosystems Services and Management Program, Laxenburg, Austria. E-mail: ermol@iiasa.ac.at for correspondence. Wolf Reuter is also at the Vienna University of Economics and Business, Vienna, Austria. The authors would like to thank David Harvey, Editor-in-Chief, and anonymous referees for important comments and suggestions which led to the improvement of this article. The development of stochastic GLOBIOM contributes to ECONADAPT (603906), TRANSMANGO (613532), and AGRICISTRADE (612755) EU FP7 projects.
} 
risk measures. A Value of Stochastic Solutions is calculated to illustrate the benefits of the stochastic over the deterministic model approach.

Keywords: Extreme events; food-energy-water-environment security; Global and Regional Interdependent land use systems; robust solutions; stochastic optimisation; strategic and adaptive decisions; systemic risks; yield shocks.

\section{JEL classifications: C61.}

\section{Introduction}

Globalisation and increasing interdependencies among land use systems (LUS) at national and international levels substantially affect the vulnerability of those systems. The interdependencies resemble a complex network connected through demand-supply relationships such that the disruption of one - perhaps due to a yield shock in one region - may catalyse systemic risks and affect LUS worldwide (OECD, 2003; Headey, 2010; Grain, 2013).

Although integrated land use planning is critically important (Arrow and Fisher, 1974; Stiglitz, 1974), the past shows that LUS policy design and implementation frequently do not account for the interdependencies and risks inherent in them. Often, the systems are governed by independent policies, and the impact of each policy on other systems is inadequately considered, if at all (e.g. Gielen et al., 1998). Multiple studies and decision support models for land use planning often rely on deterministic scenario analysis (see Gielen et al., 1998, 2000; McCarl and Schneider, 2000; Agarwal et al., 2002; Kok and Winograd, 2002), which reduces models with variable stochastic parameters to a set of scenario-specific deterministic models. This may lead to erroneous policy implications ${ }^{2}$ with highly irreversible consequences, lock-in states of development (USDE, 2008; Leclère et al., 2014), and raise critical issues for food, energy, water and environmental security (FEWES) (FAO, 2009; Headey, 2010; FAO et al., 2011). We define security as the ability to deal with risks and uncertainties to assure the necessary supply of food, feed, water, land and environmental quality under all circumstances without a substantial cost increase (Ermoliev and von Winterfeldt, 2012).

The intensifying interdependencies and vulnerability of LUS on the one hand and the need to address FEWES on the other are becoming increasingly important, and raise considerable methodological challenges. Unfortunately, the risks in interconnected natural and anthropogenic systems are analytically intractable. In contrast to standard risks, they are dependent on the decisions of various agents (OECD, 2003), which restricts traditional risk assessment and prediction. The scenario-by-scenario analysis of alternatives can provide only a range of scenario-dependent answers; they do not give a clue as to the decisions that ensure mutual stability of the systems, irrespective of which scenario applies. Therefore, in the presence of inherent uncertainties, the main issue is about designing optimal robust solutions (Ermoliev and Hordijk, 2003) that leave systems better-off under all potential scenarios. As the

\footnotetext{
${ }^{2}$ For example, the conversion of wetlands that previously served as protection substantially contributed to severe floods caused by hurricane Katrina (http://www.teenink.com/hot_topics/environment/article/297710/Causes-and-Effects-of-Hurricane-Katrina/; http://en.wikipedia.org/ wiki/Hurricane_Katrina).
} 
variety of, and the interconnections between LUS increase, the design of robust solutions has to be based on the analysis of complex systemic interactions and the risk exposures evaluated (section 3) with respect to FEWES targets.

We develop a stochastic Global Biosphere Management Model (GLOBIOM; Havlík et al., 2011) to produce integrated and robust LUS management solutions under systemic risks in a way that accounts for interdependencies among world regions. The model incorporates stochastic crop yield shocks, which facilitates the analysis of systemic risks affecting crop production and food, energy and water provision. The risks are measured in terms of regionally and globally expected shortages (or expected shortfalls) that require additional decisions, for example, on storage. Grain storage capacities, similar to global and regional insurance, reinsurance and catastrophe funds (e.g. GFDRR, 2015; Swiss Re, 2013), can increase regional and global FEWES if extreme events occur.

The structure of the paper is as follows. Section 2 presents the main motivations for developing stochastic GLOBIOM. As GLOBIOM is a large-scale, recursive-dynamic, partial-equilibrium, price-endogenous model, this section analyses its stylised fragment, demonstrating that systemic risks are characterised by the structure of the whole model, including the distribution of risks shaped by decisions, and also by the security constraints. Systemic interconnectedness (e.g. through markets or food chains or through producers of a certain commodity) is often considered beneficial. However, it can increase the vulnerability to shocks if a vital component is damaged and no alternative is readily available. Thus, using two regions as an example, section 2 shows that, unlike degenerated solutions of scenario-dependent deterministic models stressing the role of efficient regions, the stochastic GLOBIOM model calls for proper risk diversification between various kinds of region. Section 2 also illustrates that explicit treatment of uncertainties and security constraints through a two-stage stochastic optimisation (STO) model induces risk aversion in the form of Value-at-Risk (VaR) risk functions. The twostage STO distinguishes two types of decisions: strategic and adaptive. Strategic decisions (land management, storage capacities) can be viewed as decisions in the face of uncertainties (before the exact state of nature is learned). Adaptive (operational) decisions (trading, demand, price and storage withdrawals) are executed when additional information on uncertainties is revealed (after learning), allowing the policies to be adjusted. Thus, the model derives a robust combination of complementary mitigation and adaptation decisions. Ideas from this section are used for the general model in section 3 .

Section 3 concentrates on important methodological aspects of stochastic GLOBIOM using the general formulation of the model. In particular, the section characterises global multidimensional systemic risks and security criteria. A more detailed description of the model is presented in the supplementary Appendix available online at the publisher's website. In section 4, the benefits of the robust solutions derived using the stochastic model in comparison with the solutions of its deterministic counterpart are measured using the Value of Stochastic Solution (VSS) (Birge, 1982). We illustrate the stochastic approach by examining robust storage strategies towards systemic risks by buffering production shortfalls and meeting security requirements at regional and global levels. Many models look either only at land (Veldkamp and Fresco, 1996; Verburg et al., 2000) or at storage decisions (Gustafson, 1958; Deaton and Laroque, 1996). Stochastic GLOBIOM allows both to be done within the same modeling framework. Section 5 concludes. 


\section{Systemic Risks and Security Targets}

\subsection{Deterministic GLOBIOM}

Before focusing on stochastic aspects, we provide a conceptual description of the general deterministic GLOBIOM (Havlík et al., 2011). GLOBIOM is a global recursivedynamic, partial-equilibrium model running at the level of major countries and world regions. This section illustrates the variety of decision variables and exogenous drivers (parameters), which provide the basis for the stochastic version of GLOBIOM to address systemic risks in LUS. The model integrates the agricultural, bioenergy and forestry sectors allowing for policy analysis of global and regional issues concerning land use competition and land use transformations driven by increasing demands for food, feed, water and biofuels (Coyle, 2007). GLOBIOM endogenously projects land demand and resulting changes by land use types and regions. The main land uses distinguish crop land, grass land, forest (managed and non-managed) land, fast-rotation forest plantations, and natural land. Land use change alternatives are limited by explicit food, feed, energy, water and environmental security constraints.

The supply of crops (i.e. agricultural production) needs to cover food and feed demands. The food security constraint ensures that the energy intake from food cannot be lower than the minimum amount of kilocalories needed to satisfy dietary requirements in cereals, vegetable and animal products (meat and dairy products) measured in kilocalories per capita (WHO, 1985; James and Schofield, 1990). Feed sources for livestock comprise crops, grass and biofuel co-products (feed cakes). Feeds produced for livestock cannot be lower than the minimum livestock dietary requirements in energy measured in megacalories. First-generation biofuels from crops and second-generation biofuels from lignocellulosic biomass (woody crops) and agricultural residues have to fulfill biofuel production targets. Food security constraints and biofuel security targets introduce competition for limited natural resources (land and water) among different land uses.

Forestry resources are used for the production of saw logs, pulp logs and other industrial logs. Forest production also includes biomass for woody energy and traditional fuel wood. The energy biomass can be converted through (i) combined heat and power production, (ii) fermentation for ethanol, heat, power and gas production, and (iii) gasification for methanol and heat production. Woody biomass for energy can also be produced from short-rotation tree plantations. Thus, agriculture and forestry have binding bioenergy targets (Havlík et al., 2011) which induce systemic risks, illustrated by example in section 2.2. Environmental ${ }^{3}$ security constraints are introduced as targets on GHG emissions from land use and land use changes (Valin et al., 2013).

Global interdependencies between demand, prices, international trade flows and environmental constraints are analysed in an endogenous manner for 28 world regions (Havlík et al., 2011), while decisions on production and land use allocation are taken at a $50 \times 50 \mathrm{~km}^{2}$ grid cell resolution. Product supply functions are included implicitly and are based on detailed, geographically explicit Leontieff production functions. We use the Environmental Policy Integrated Model (EPIC) model (Liu

\footnotetext{
${ }^{3}$ Discussions of environmental security have been evolving since 1970 (Myers, 1989). Several definitions of environmental security have been adopted by a few countries and international organisations. An overview of the definitions is found in The Millennium Project, http://millennium-project.org/, Myers (1989).
} 
et al., 2007) to simulate climate- and management-related yields for $20 \mathrm{crops}$, which represent more than $80 \%$ of the 2007 harvested area as reported by FAO (2009). The GLOBIOM model is formulated as a linear optimisation problem. The objective function of GLOBIOM maximises the sum of producer and consumer surpluses subject to food security, biofuel targets, GHG emissions and resource constraints. For further details on GLOBIOM, the reader is referred to Havlík et al. (2011) where all basic assumptions on exogenous drivers (i.e. population, economic, environmental and technological development parameters, etc.) are also presented in detail. In the deterministic version, crop yield variability is not taken into account in the decision variables of a social planner (i.e. the model assumes expected crop yields). This is equivalent to dealing with only one scenario of possible developments. However, averaging may lead to erroneous policy conclusions and remove the diversification necessary to manage the risks.

\subsection{Systemic risks}

Global Biosphere Management Model is a large-scale model; therefore in this section we analyse its main features with a stylised example in order to understand the main drivers of systemic risks. The objective function of stochastic GLOBIOM maximises total expected net benefit (benefits-costs) of consumers and producers under endogenously calculated prices (supplementary Appendix, equation S1, available online). In this simplified example we ignore the elasticity of the demand, reducing the model to minimising the total cost subject to a demand security constraint. We analyze systemic risks in a simple social planning model with only two regions cooperating in bio-ethanol production from corn and wheat, $i=1,2$, to meet exogenous inelastic demand $d$. Systemic risks are induced through interdependencies between decisions of regions and supply uncertainties. Corn, which is traditionally used for bio-ethanol production, provides the highest yield at the lowest cost and wheat for bio-ethanol may be used in lean corn years. Let $x_{i}$ denote the production level of region $i$, and $c_{i}$ is production unit cost. We assume that in the case of production shortage, the goods can be purchased on a market with a per unit price of $b$. Assume the first producer is the cheapest, $c_{1}<c_{2}<b$.

\subsubsection{Absence of uncertainty}

In the absence of uncertainty (i.e. using average values and ignoring variability), the deterministic model is formulated as the minimisation of the total cost function:

$$
c_{1} x_{1}+c_{2} x_{2}+b y
$$

subject to the supply-demand security constraints:

$$
x_{1}+x_{2}+y \geq d, \quad x_{1} \geq 0, x_{2} \geq 0 .
$$

The optimal solution to the problem is $x_{1}^{*}=d, x_{2}^{*}=0, y^{*}=0$, that is, the degenerated solution of the deterministic model (1)-(2) leaves all production to the most efficient region.

\subsubsection{Systemic risk, cooperation, and risk sharing}

Consider the more realistic situation of planning production under uncertainty due to yield variability, which may reduce production $x_{1}, x_{2}$. In this case, the endogenous supply (2) is transformed to a constraint: 


$$
a_{1}(\omega) x_{1}+a_{2}(\omega) x_{2}+y(\omega) \geq d,
$$

where $a_{1}(\omega)$ and $a_{2}(\omega)$ are random shocks to $x_{1}, x_{2}$, for example, due to weather variability, $0 \leq a_{i}(\omega) \leq 1, i=1,2$. We do not specify the structure of uncertain events $\omega$, which may affect both regions simultaneously or independently. In general, we can think of a vector $\omega=\left(\omega_{2}, \omega_{2}\right)$, where $\omega_{1}$ and $\omega_{2}$ can be dependent random variables (e.g. yield shocks), simulated by a Monte Carlo model producing a sequence of potential scenarios $\omega=\omega^{s}$, for example, weather events, $s=1,2, \ldots$, with components $\omega_{i}^{S}, i=1,2$, which are then used in the EPIC model to calculate the yields shocks $a_{1}(\omega)$ and $a_{2}(\omega)$. Often, $\omega$ is characterised by a finite number of scenarios (Kall and Mayer, 2004). Decisions on production $x=\left(x_{1}, x_{2}\right)$ at stage 1 have to be made before observing exact values $a_{1}(\omega)$ and $a_{2}(\omega)$. If supply $a_{1}(\omega) x_{1}+a_{2}(\omega) x_{2}$ falls short of demand $d$, the residual amount $d-a_{1}(\omega) x_{1}-a_{2}(\omega) x_{2}$ must come from purchasing $y$ $(\omega)$ on the market at stage 2 at per unit price $b$. The concept of two-stage modeling with strategic (ex-ante) $x_{1}, x_{2}$ and adaptive (ex-post) decisions $y(\omega)$ is critically important for robust land use planning, as these decisions ensure the security constraint (3) for any event $\omega$. The deterministic model (1)-(2) is now formulated as a linear twostage STO model: minimise the cost function

$$
c_{1} x_{1}+c_{2} x_{2}+b E y(\omega)
$$

subject to security constraint (3) for all potential scenarios of uncertainty $\omega$, where $E y$ $(\omega)$ is the expected value of the shortfall $y(\omega)$, that is, $E y(\omega)=\sum_{s} p_{s} y\left(\omega^{s}\right)$, for discrete scenarios $\omega^{s}$ and their probabilities $p_{s}, s=1,2, \ldots$ In this simple example, the optimal stage 2 decision $y(x, \omega)$ satisfying constraint (3) for any fixed vector $x=\left(x_{1}, x_{2}\right)$, $x_{1} \geq 0, x_{2} \geq 0$ and $\omega$ can be found analytically as $y(x, \omega)=\max \left\{0, d-a_{1}(\omega)\right.$ $\left.x_{1}-a_{2}(\omega) x_{2}\right\}$. Therefore, optimal strategic decisions $x_{1}$ and $x_{2}$ minimising function (4) also minimize:

$$
F(x)=c_{1} x_{1}+c_{2} x_{2}+b \operatorname{Emax}\left\{0, d-a_{1}(\omega) x_{1}-a_{2}(\omega) x_{2}\right\},
$$

where $\operatorname{Emax}\left\{0, d-a_{1}(\omega) x_{1}-a_{2}(\omega) x_{2}\right\}$ is the expected shortfall characterising the systemic risks and vulnerability of the supply $x_{1}, x_{2}$.

It is important to emphasise that the model (5) does not directly include any measure of risk in the objective function. The risk aversion arises through the coexistence of ex-ante $x$ and ex-post $y(x, \omega)$ decisions in the form of VaR quantile-based risk constraint (Ermoliev and Jastremski, 1979; Ermoliev and Norkin, 1997). Let us show that robust $x_{1}^{*}, x_{2}^{*}$ satisfy quantile-based risks constraints induced by interdependencies between uncertainties $a_{1}(\omega)$ and $a_{2}(\omega)$, decisions $x=\left(x_{1}, x_{2}\right)$, security requirements (3), and the costs. Assume that only the efficient region is at risk (i.e. $a_{2}=1$ ). Region 2 may be viewed as an inefficient region $\left(c_{1}<c_{2}\right)$. Yet, we shall see that this region, due to the interdependencies, is the key player in terms of securing the supply. Let function $F(x)$ have continuous derivatives; for example, the distribution function of $a_{1}(\omega)$ has a continuous density. This assumption avoids the use of more sophisticated nonsmooth STO techniques (Ermoliev and Wets, 1988). The optimal positive solution $x_{1}^{*}>0, x_{2}^{*}>0$ exists in the case when $F_{x_{1}}(0,0)=c_{1}-b E a_{1}(\omega)$ and $F_{x_{2}}(0,0)=c_{2}-b$ are negative, where $E a_{1}(\omega)$ is the expected value of $a_{1}(\omega)$. The efficient region 1 is inactive in the case $c_{1}-b E a_{1}(\omega)>0$, leaving production entirely to region 2. Both regions are active only in the case when $c_{1}-b E a_{1}(\omega)<0$. Somewhat surprisingly, inefficient region 2 is active unconditionally because $c_{2}-b<0$. 
It is important to derive the optimal production level $x_{2}^{*}>0$ of the inefficient producer. Using optimality conditions of type $F_{x_{2}}(x)=0$ for stochastic minimax models (see Ermoliev and Wets, 1988; Ermoliev and Norkin, 1997), it is defined by the equation:

$$
\operatorname{Prob}\left(d-a_{1}(\omega) x_{1}^{*} \geq x_{2}^{*}\right)=c_{2} / b
$$

that is, the optimal production $x_{2}^{*}$ of the risk-free region 2 is a quantile of the probability distribution characterising the contingencies $a_{1}(\omega)$ of region 1. Equation (6) shows that the ex-ante first-stage decisions $x^{*}$ cover only a fraction of the risks determined by the quantile $c_{2} / b$, whereas second-stage decisions hedge the rest of the exposure (i.e. the production shortfall). Although not at risk, region 2 is affected by systemic risks characterised by the structure of the whole supply system, that is, demand $d$, shocks $a_{1}(\omega)$, cost function $c_{2}$, import prices $b$, robust decisions $x_{1}^{*}$ and $x_{2}^{*}$, and the security constraint (3). These risks can be regulated by adjusting parameters $c_{1}$ and $c_{2}$ on local (regional) and $b$ and $d$ on global (national and international) levels. In engineering, insurance, and financial applications, equations of type (6) are known as chance (or probabilistic) constraints (Prekopa, 1988), safety or reliability constraints (Marti, 2008), or VaR constraints (Rockafellar and Uryasev, 2000). The optimal value $F\left(x^{*}\right)$ is a Conditional Value-at-Risk or CVaR risk measure (Rockafellar and Uryasev, 2000).

\section{Stochastic GLOBIOM}

\subsection{A two-stage model}

In this section, similar to model (3), (4), we use the two-stage approach to formulate general stochastic GLOBIOM. The model involves uncertainty and risks associated with different scenarios $\omega=\left(\omega^{1}, \omega^{2}, \ldots\right)$ of potential crop yields. Accounting for direct and indirect dependencies among 28 world regions, stochastic GLOBIOM allows robust joint solutions to be identified with respect to production, trade, price and storage to ensure food and feed security goals, bio-ethanol production targets and resources and $\mathrm{CO}_{2}$ emission constraints from LUS in all regions, under all scenarios $\omega$. As illustrated by the example in section 2, the variability of yields induces systemic risks, which may cause non-risky activities to become indirectly risk-exposed. First-stage strategic decisions are taken before the actual yield is observed and do not depend on scenarios $\omega$. We denote them collectively by a vector $x$. These decisions include crop acreage and storage capacities. Second-stage adaptive decisions $y(\omega)$ include trade choices, storage withdrawals, demand and prices, and these are made after the yield scenario is observed.

The general stochastic version of GLOBIOM is formulated as a two-stage problem of STO (programming) (Ermoliev and Wets, 1988; Birge and Louveaux, 1997): maximise with respect to decision variables $(x, y(\omega))$ the function:

$$
F(x)=E_{\omega} f(x, y(\omega), \omega)=\int f(x, y(\omega), \omega) P(d \omega)
$$

under constraints:

$$
g_{i}(x, y(\omega), \omega) \leq 0, \quad i=\overline{1, m},
$$

where the goal function $F(x)$ represents total systemic welfare or net benefits associated with both types of decision $(x, y(\omega))$. Functions $g_{i}(x, y(\omega), \omega)$ denote various 
security constraints and performance indicators, and vector $\omega$ denotes potential scenarios of yields. A detailed description of functions $F$ and $g_{i}$ can be found in the supplementary Appendix, available online at the publisher's website.

In numerical applications, the model (7), (8) is often formulated (Ermoliev and Wets, 1988; Birge and Louveaux, 1997; Kall and Mayer, 2004) using a finite set of explicitly or implicitly given scenarios $\omega^{s}, s=1, \ldots, S$ : maximise:

$$
\sum_{s=1}^{S} p_{s} f\left(x, y^{s}, \omega^{S}\right)
$$

subject to:

$$
g_{i}\left(x, y^{s}, \omega^{s}\right) \leq 0, \quad i=\overline{1, m}, \quad s=\overline{1, S},
$$

where $\omega^{1}, \omega^{2}, \ldots, \omega^{S}$ is a sample of scenarios $s=\overline{1, S}$ for uncertain vector $\omega$ derived from real observations, uncertainty generators (e.g. EPIC model), or/and expert opinions, and $y^{s}$ denotes scenario-specific second-stage decisions $y\left(\omega^{s}\right)$. Probabilities $p_{1}$, $\ldots, p_{S}, \sum_{s=1}^{S} p_{S}=1$, can be calculated from historical data, suggested by experts or generated by models. In the absence of any information, probabilities $p_{s}, s=\overline{1, S}$, can be uniformly distributed with $p_{s}=1 / S$ or defined by feasible sets such as $p_{1} \leq p_{2}$, $p_{2}+p_{5} \geq p_{3}$, and so on.

\subsection{Security criteria}

The model (9)-(10) assumes the feasibility of choosing strategic and adaptive decisions $\left(x, y^{s}\right)$ controlling constraints (10) for any scenario $\omega^{s}$ from the admissible set. Extreme weather-related events or market prices may affect large territories and the structure of LUS (i.e. functions $f$ and $g$ ). For example, droughts may restrict trading capacity with some regions. Therefore, in general, the constraints of model (9)-(10) can be fulfilled only for some scenarios (i.e. with a certain probability). In this case, we can think about a 'lack' of systemic security characterised by a set of decision variables $z_{i s} \geq 0$ satisfying equations:

$$
g_{i}\left(x, y^{s}, \omega^{s}\right) \leq z_{i s} .
$$

Variables $z_{i s}$ are viewed as regional (and global $\sum_{i} z_{i s}$ ) systemic shortages (shortfalls) requiring additional decisions such as storages, investments, insurance, financial instruments and other options. There is an essential difference between ex-post decisions $y^{s}$ and $z_{s}$. Decisions $y^{s}$ can be viewed as already existing measures (e.g. possible trading connections), whereas $z_{s}$ are additional capacities (e.g. grain, water, energy storages) that must be constructed in proper locations (regions, countries, grids) to guarantee a desirable regional and global systemic security level. Accounting for possible new capacities $z_{s}$, let us consider the maximisation of the function:

$$
\sum_{s=1}^{S} p_{s} f\left(x, y^{s}, \omega^{s}\right)-\sum_{s=1}^{S} p_{s}\left(\pi_{s}, z_{s}\right),
$$

subject to security constraints $(11)$, where $\pi_{s}=\left(\pi_{1 s}, \ldots, \pi_{m s}\right) \geq 0, z_{s}=\left(z_{1 s}, \ldots, z_{m s}\right)$, $s=1, \ldots, S$, are scaling vectors or security weights, risk premiums for insurance, price of contingent credits, etc.; $(\cdot, \cdot)$ denotes the scalar product of vectors. The set of all $x, y^{s}, s=1, \ldots, S$, satisfying equation (11) for $z_{s}=0, s=1, \ldots, S$, can be identified as the security set. If optimal solution $x^{*}, y_{s}^{*}, z_{s}^{*}, s=1, \ldots, S$, of model (11), and (12) has some $z_{s}^{*} \neq 0$, then equations (10) are satisfied only for some scenarios. 
Random variable $\pi_{i s} z_{i s}^{*}$ characterises systemic risk associated with violation of security constraint (10), whereas random variable $\sum_{i} \pi_{i s} z_{i s}^{*}$ characterises total (global) systemic risk. Therefore, criterion (12) provides a trade-off between the social welfare defined by the first criterion and the systemic security (risks). Security constraints in the model (11)-(12) are regulated by parameters $\pi_{s}$. Larger $\pi_{s}$ impose stronger security requirements. These constraints can be introduced in the form of probabilistic constraints (Ermoliev et al., 2000b) similar to insolvency constraints (Colin and Kunreuther, 1993; Ermoliev et al., 2000a) in insurance or reliability (safety) constraints (Marti, 2008) in engineering:

$$
P\left\{\omega: g_{i}(x, y(\omega), \omega)-g_{i}^{*} \geq 0\right\} \geq \gamma_{i},
$$

where $g_{i}^{*}$ is a targeted level of indicator $g_{i}(x, y(\omega), \omega)$, for example, minimum (or recommended) food and feed requirements and bioenergy goals (see equations S2.1, S2.2 and S3 in the supplementary Appendix, available online at the publisher's website). The right-hand-side parameter $\gamma_{i}$ imposes the desirable security level specifying the probability of fulfilling the constraint (13). The probabilistic constraints define a nonconvex and possibly highly discontinuous optimisation model requiring specific solution methods. Therefore, they are often substituted (see e.g. Ermoliev et al., 2000b) by the following risk functions:

$$
\operatorname{Emax}\left\{0, g_{i}(x, y(\omega), \omega)-g_{i}^{*}\right\}
$$

characterising supply shortages (shortfalls). In this case, vector $z_{s}=\left(z_{1 s}, \ldots, z_{m s}\right)$ in (12) is given explicitly as $z_{i s}=\max \left\{0, g_{i}\left(x, y^{s}, \omega^{s}\right)-g_{i}^{*}\right\}$ and the problem (11)-(12) is reformulated as maximising:

$$
\sum_{s=1}^{S} p_{s} f\left(x, y^{s}, \omega^{s}\right)-\sum_{i=1}^{m} \sum_{s=1}^{S} p_{s} \pi_{i s} \max \left\{0, g_{i}\left(x, y^{s}, \omega^{s}\right)-g_{i}^{*}\right\}
$$

or equivalently as maximising function (12), that is:

$$
\sum_{s=1}^{S} p_{s} f\left(x, y^{s}, \omega^{s}\right)-\sum_{s=1}^{S} p_{s}\left(\pi_{s}, z_{s}\right)
$$

under constraints:

$$
z_{i s} \geq g_{i}\left(x, y^{s}, \omega^{s}\right)-g_{i}^{*}, \quad z_{i s} \geq 0, \quad i=\overline{1, m}, \quad s=\overline{1, S} .
$$

The most important factor for real applications is that this approach converts discontinuous constraints (13) into a linear optimisation problem (16)-(17) similar to deterministic GLOBIOM.

\subsection{Global systemic risks and security}

The proposed general stochastic model integrates 28 world regions. It has a rich set (see section 2.1, and the supplementary Appendix, available online) of decision variables (internal drivers) and parameters (external drivers), providing unique possibilities for analysing and managing regional and global systemic risks affecting various interdependent LUS. These risks are associated with a violation of the security constraints (10), (11) regulated by vector $z_{s}$ and parameter $\pi_{s}$ in the objective function (12).

The following shows that, similar to the simplest two-region model in section 2.2, the interdependent global systemic risks in general stochastic GLOBIOM are defined 
by a system of quantile-based multidimensional equations of type (6), (13). Consider the model (7), (8) with linear stochastic functions:

and

$$
f(x, y(\omega), \omega)=\sum_{j} c_{j} x_{j}+\sum_{k} d_{k}(\omega) y_{k}(\omega)
$$

$$
g_{i}(x, y(\omega), \omega)=\sum_{j} a_{i j}(\omega) x_{j}+\sum_{k} b_{i k}(\omega) y_{k}(\omega)+e_{i}(\omega),
$$

where $g_{i}$ stands for various systemic security indicators, and $c_{j}, d_{k}(\omega), b_{i k}(\omega), e_{i}(\omega)$ are deterministic and stochastic parameters. The model (15) can be formulated using the general type of scenarios $\omega$ as maximising adjusted to risk expectation function:

$$
F(x)=E\left[f(x, y(\omega), \omega)-\sum_{i=1}^{m} \pi_{i}(\omega) \max \left\{0, g_{i}(x, y(\omega))-g_{i}^{*}\right\}\right] .
$$

Similarly to section 2.2 , let us consider a positive component $x_{j}^{*}>0$ of the optimal solution $\left(x^{*}, y^{*}(\omega)\right)$ maximising $F(x)$. Again, for simplicity of illustration, assume that $F(x)$ has continuous partial derivative $F_{x_{j}}\left(x^{*}\right)$ and that $a_{i j}(\omega), \pi_{i}(\omega)$ are deterministic parameters $a_{i j}, \pi_{i}$. Using formulas for gradients of stochastic maximin functions (Ermoliev and Norkin, 1997) we obtain the equation for systemic risk equilibrium: if $x_{j}^{*}>0$, then:

$$
F_{x_{j}}\left(x^{*}\right)=c_{j}-\sum_{i=1}^{m} \pi_{i} a_{i j} \operatorname{Prob}\left[g_{i}(x, y(\omega), \omega) \geq g_{i}^{*}\right]=0 .
$$

Thus, global systemic risk indicators $\operatorname{Prob}\left[g_{i}(x, y(\omega), \omega) \geq g_{i}^{*}\right]$ and the respective robust combination of ex-ante and ex-post solutions $x, y(\omega), z(\omega)$ are derived, with stochastic GLOBIOM (20) solving a system of linear equations, with respect to these indicators, (21). It is remarkable that, as in section 2.2, these computations avoid direct evaluations of underlying probability distributions. Yet, it is complicated to derive the indicators and the solutions in an analytical form, as in section 2.2. Therefore, we consider some numerical results of stochastic GLOBIOM: in particular, histograms of global storage withdrawals $\sum_{i} z_{i s}^{*}$ hedging regional and global systemic risks, which can be fine-tuned by parameter $\pi_{s}$ to a desirable level.

\section{Numerical Results}

This section analyses the advantages of robust solutions derived with stochastic GLOBIOM vs. solutions derived using traditional scenario analysis of its deterministic counterpart. The two-region stochastic model in section 2.2 illustrates the essential differences between these solutions. In particular, somewhat surprisingly, the inefficient region 2 (e.g. more labour- than capital-intensive), that is inactive in the deterministic case, becomes unconditionally active in the stochastic model, enabling robust production to fulfill biofuel security constraints. We calculate the 'VSS', which is used to measure the importance of applying the stochastic model (Birge, 1982).

\subsection{Data}

Using data from 1960 to 2012 made available by the Food and Agriculture Organization (http://faostat.fao.org) we derive yield probability distributions to model yield 
shocks according to different regions and crops. The analysis follows the approach used in Beach et al. (2010). The data have been detrended and normalised by 2012 yield. As an example, histograms (i.e. empirical probability distributions) of wheat yield based on a 52-year data series are depicted in Figure 1 for selected countries.

Yield distributions are characterised by multimodal shapes which precludes the use of mean-variance criteria and indicators (Markowitz, 1952). Figure 1 also shows the main statistics (i.e. average and main -5 th, 50th and 95 th - percentiles) of yield distributions. Countries such as France and China are characterised by higher yields and smaller yield variability. In Russia, US and Brazil, the yields are smaller and the variability is larger.

\subsection{Robust solutions: Strategic and operational decisions}

In this paper, robust solutions of stochastic GLOBIOM comprise strategic decisions (land allocation by LUS and storage capacities) and related adaptive decisions (trade,
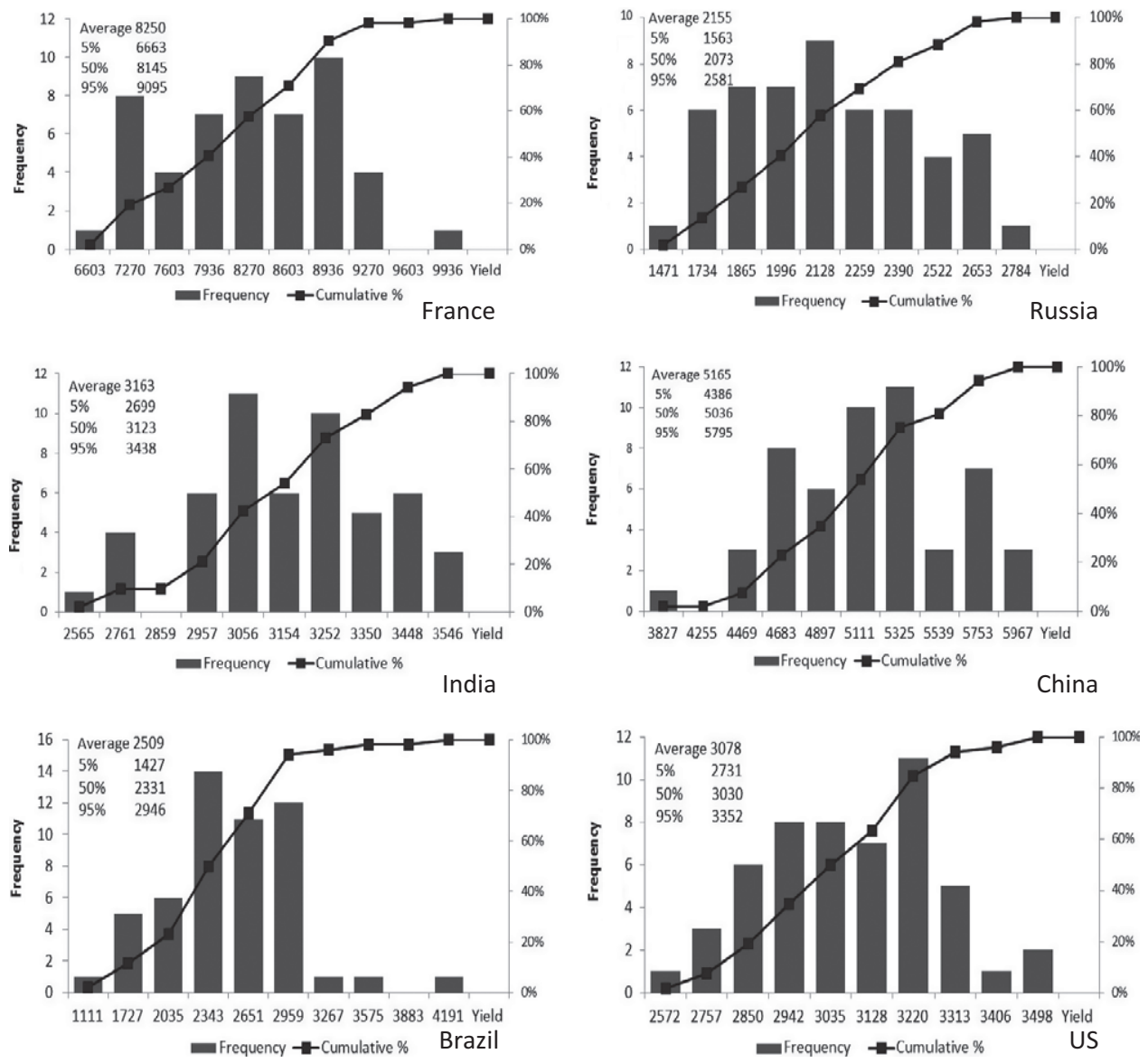

Figure 1. Empirical wheat yield distribution by selected grain producers, 1960-2012 Notes: Horizontal axis denotes yield (in kilograms per hectare of harvested land) and vertical axis shows the number of years (frequency) the corresponding yield occurred in the 1960-2012 period. Cumulative distribution refers to the percentage of total of the yield occurrences at or below the value on the horizontal axis.

Source: FAO, http://faostat.fao.org. 
storage withdrawals, prices, demands). Stochastic GLOBIOM runs for a time horizon of 40 years (from 2010 to 2050) with a time step of 10 years. Stochastic yields are represented by a finite set of historical yield scenarios from 1960 to 2015, analysed in section 4.1. In the calculations, we use food, feed, water, environmental and biofuel security constraints by requiring that, in each yield scenario, the demand for food, feeds and biofuels is not less than the exogenously given targeted levels, as in equation (13) and functions (14). Water security is introduced through a constraint on the total admissible water consumption by the following activities: crop production and processing, animal farming, forest production and processing, and biofuel production and conversion. GHG emissions targets from LUS (Valin et al., 2013) are included as environmental security constraints.

The benefits of robust solutions derived with stochastic GLOBIOM over the solutions of its deterministic counterpart are measured with the VSS. The VSS is calculated by comparing the value of the stochastic goal function $F\left(x^{* \text { sto }}\right)$ (equation $\mathrm{S} 1$ in the supplementary Appendix, available online) using the robust $x^{* \text { sto }}$ solution with the value of the stochastic goal function $F\left(x^{* \text { det }}\right)$ using the deterministic $x^{* \text { det }}$ solution. The value $F\left(x^{* \text { sto }}\right)$ is by about $25 \%$ higher than $F\left(x^{* \text { det }}\right)$, indicating the gains from using the stochastic model.

We analyse two cases of policy recommendations. The first case $(\mathrm{C} 1)$ represents a popular approach to examining uncertainty and risks through a scenario analysis; that is, deterministic GLOBIOM is run in a 'what-if' manner, using alternative yield scenarios. In each scenario, the model provides scenario-specific recommendations. The second case (C2) involves stochastic GLOBIOM and corresponds to planning under uncertainty and risk when strategic (ex-ante) decisions on land allocation between LUS are made before information on stochastic yields becomes available. These decisions are adjusted using adaptive (ex-post) decisions (trade, storage withdrawals, prices) after the actual yield is observed, thus ensuring robustness of coordinated strategic and adaptive decisions. The combination of the decisions minimises the costs of implementing the strategic decisions and the costs of adjustment actions in response to each yield shock propagating through LUS.

In case (C1), GLOBIOM assumes that a spatio-temporal yield scenario occurs with probability 1 , and there is no need to adjust to shocks. When all is known in advance, LUS can manage without ex-post responses. In each yield shock scenario, the global commodity market redistributes production 'shortages' between regions so that food, energy, water and environmental constraints are satisfied. However, the implementation of these scenario-dependent solutions can require considerable adjustments, such as conversion of forest into crop land or additional irrigation capacities if, for example, a drier than anticipated year occurs. Figure 2 presents percentage of land in different LUS in $\mathrm{C} 1$ and $\mathrm{C} 2$ cases at the global level.

To fulfill FEWES targets in the face of all yield shocks, stochastic GLOBIOM suggests that crop land be used only $0.1 \%$ more compared with the results of deterministic GLOBIOM (C1) under the average yield scenario (Figure 2, panel a). In some yield scenarios, for example, those in the year 2000 when droughts occurred simultaneously in Australia, Russia and China (Zou et al., 2005; Spinoni et al., 2015), the actual demand for crop land may exceed the robust requirement (demand) for crop land calculated using stochastic GLOBIOM.

Having the possibility of flexible ex-post adjustments to all potential scenarios, stochastic GLOBIOM recommends qualitatively different solutions. For example, natural ecosystems should be preserved, the conversion of natural forests into 
(a) Crop land

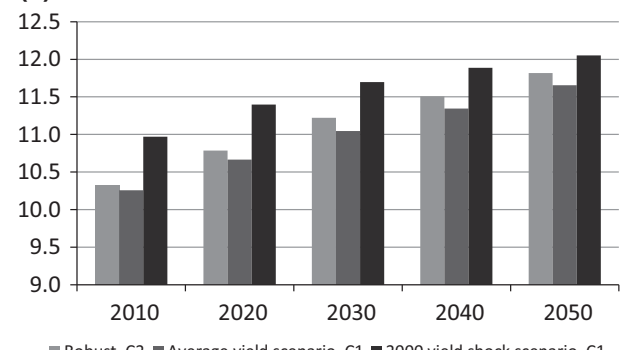

(c) Natural forest

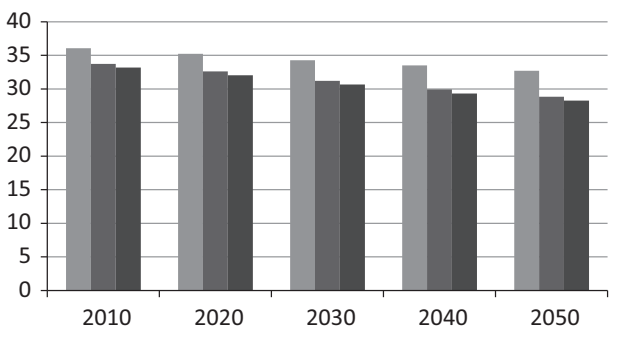

— Robust, C2 $\square$ Average yield scenario, C1 $\square 2000$ yield shock scenario, C1

(e) Planted forest

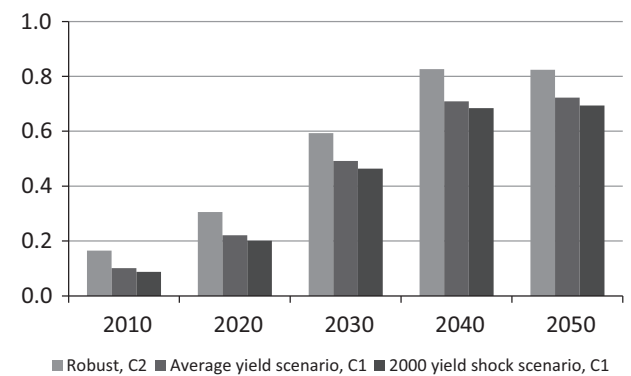

(b) Grass land

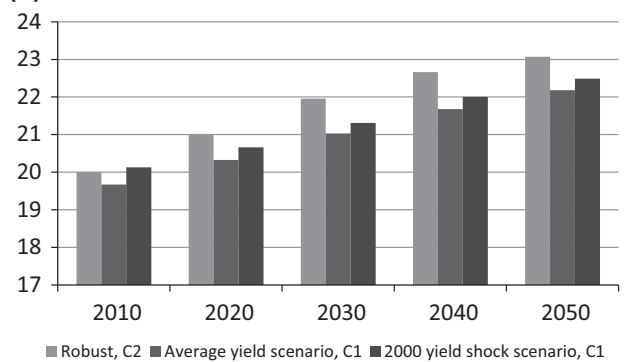

(d) Managed forest

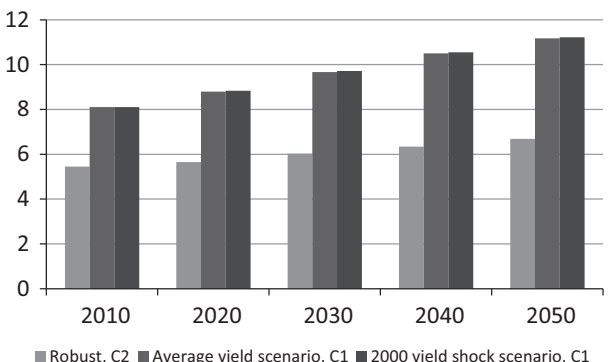

(f) Natural land

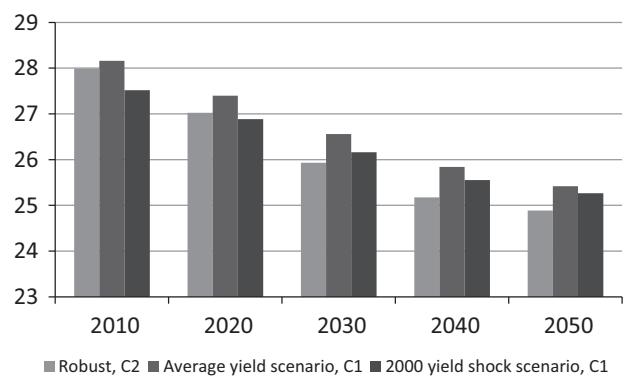

Figure 2. Percentage of total land occupied by different LUS calculated using stochastic GLOBIOM (Robust, C2), deterministic GLOBIOM under the average yield scenario (Average yield scenario, C1), and deterministic GLOBIOM under extreme shock scenario (2000 yield shock scenario, C1). Horizontal axis labels simulation year and vertical axis identifies percent. (a) Crop land; (b) Grass land; (c) Natural forest; (d) Managed forest; (e) Planted forest; (f) Natural land.

managed woodland should slow down, grass land should be protected as an important feed source for livestock (see panels b and c in Figure 2). At the same time, it calculates a higher percentage of short-rotation tree plantations to fulfill bioenergy goals (Figure 2, panel e). All conversions come primarily from natural land (Figure 2, panel f). It is critically important that robust strategic decisions on land allocation among LUS are supplemented with adaptive scenario-specific trade decisions $y^{s}$ in the objective function (12). Stochastic GLOBIOM accounts for spatial dependencies between yield shocks (FAO, 2011) and suggests scenario-specific geographical diversification of trade across uncorrelated (or negatively correlated) regions and commodities. 
To hedge global systemic risks and ensure FEWES goals, stochastic GLOBIOM makes use of storage facilities (which in the deterministic case are not required) to hedge regional and global (systemic) risks of shortfalls. They are essential for self-sufficient management of interdependent systemic risks to cover food demand when trading is restricted or limited because of a direct or indirect (induced) yield and price shocks, or when land and water resources are scarce. Thus, storage can be viewed as insurance in cases where no other sources of supply, domestic or foreign, are available. In this sense, storage capacities measure the systemic risks and (in)security, as discussed in section 3.3. From the formal point of view, storage withdrawals correspond to decision variables $z_{i s}$ in (12) and (21). By changing $\pi_{i}$, the stochastic model can achieve more or less systemic risk hedging. Adjustments of storage can be introduced to help ensure the required global FEWES levels, as discussed in section 3.3, equation (21).

Our calculations using stochastic GLOBIOM show that stochastic yields induce considerable volatility of global prices affecting various regions (Wright, 2011). This reduces the robustness of trade-based systemic risk management. Therefore, robust solutions of stochastic GLOBIOM in the presence of grain storage can increase the feasibility of biofuel targets, as discussed in section 2.2. For example, storage capacities (Figure 3) of about 80 and 300 thousand tons for rape and sunflower, respectively, modulate the instantaneous demand for crop land caused by a yield shock (similar to the year 2000, as in Figure 2, panel a), and decrease the investments in and conversion of rainfed land into irrigated land to sustain rare high-impact shocks. Global reserves (Figure 3) of about 1,000, 250, 80 and 300 thousand tons of rice, barley, wheat, rape, sunflower, respectively, can obviate the need for investment in the irrigation of about 3,545 thousand hectares of agricultural land globally.

The availability of storage decreases prices and stimulates the increase in demand for commodities produced in interdependent LUS and necessary for FEWES. Figure 4 compares global demand for selected crops. For example, stochastic GLO$\mathrm{BIOM}$ allows that rice and wheat demand be increased by about $4.5 \%$ and $6 \%$, respectively, compared with the deterministic model. On the other hand, the model suggests that production of rape and sunflower be decreased by about $5 \%$ and $6 \%$, respectively. Stochastic GLOBIOM also recommends that biofuel targets can be fulfilled at lower cost through using cheaper biofuel feedstocks, e.g. second-generation biofuels from lignocellulosic biomass produced on short rotation tree plantations (Figure 1, panel e).

\section{Concluding Remarks}

The paper develops the stochastic GLOBIOM model, accounting for interdependencies among the main LUS on the global, national, and grid-cell levels. As section 2 demonstrates, shocks due, for example, to weather variability, may induce systemic risks that implicitly affect non- risky regions and activities. Analysis of FEWES in LUS under these 'distributed' risks requires robust solutions that comprise a proper set of ex-ante strategic (first-stage) and ex-post adaptive (second-stage) decisions enabling flexible adjustments to be made when new information becomes available. Thus, the ex-ante first-stage decisions cover only a fraction (quantile) of the risks determined by the FEWES requirement, whereas second-stage decisions hedge the rest of the exposure. 
(a) Rice.

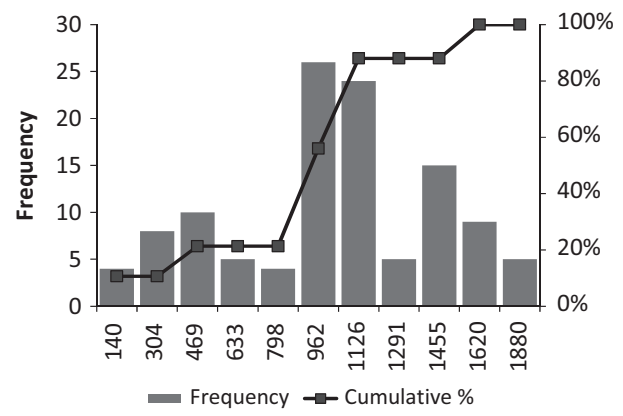

(c) Rape.

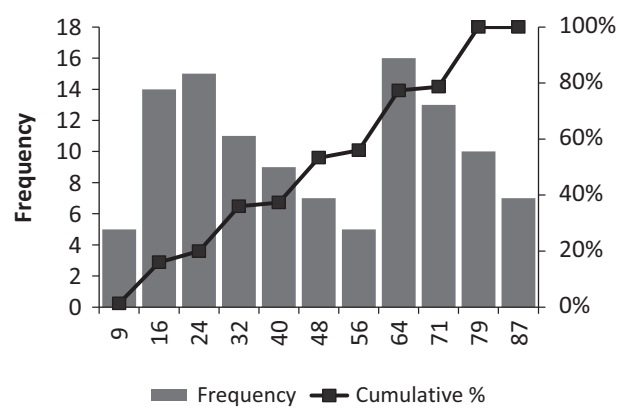

(b) Wheat.

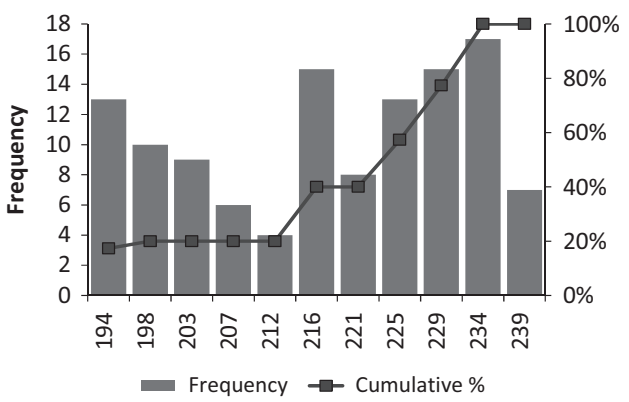

(d) Sunflower.

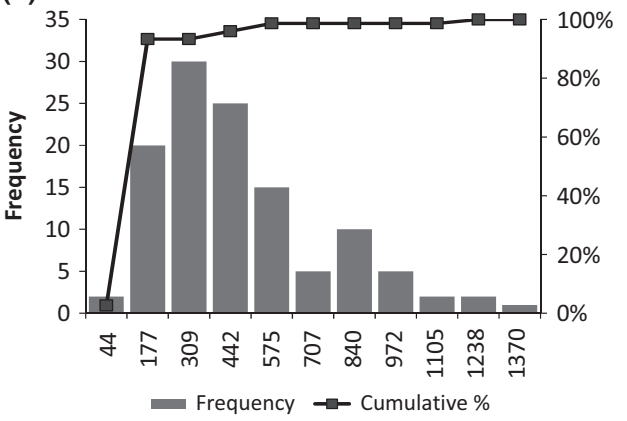

Figure 3. Distribution of storage withdrawals, in thousand tons, at the global level. Frequency refers to the absolute number of withdrawals within a range identified on the horizontal axis. Cumulative refers to the percentage of total withdrawals at or below the value on the horizontal axis. (a) Rice; (b) Wheat; (c) Rape; (d) Sunflower.

The risks in general stochastic GLOBIOM are defined by a system of quantilebased multi-dimensional security equations characterising production and resource shortfalls (shortages) at regional and global level (section 3). Robust management of shortfalls requires additional decisions to be made, for example, such as grain, water and energy stores, where storage capacities are similar to global and regional catastrophe funds. In section 4 we show that stochastic GLOBIOM calculates robust grain and oilseed storage, which, in the presence of yield shocks and resource constraints help to fulfill combined FEWES targets (e.g. biofuel security) at lower costs without diverting grains from human consumption. By proper risk-adjusted spatial redistribution of production and selection of management strategies and processing technologies, stochastic GLOBIOM suggests lowering production of biofuel crops such as rape and sunflower and instead making use of cheaper feedstocks such as lignocellulosic biomass from short rotation tree plantations. Analysing grain and oilseed storage is essential for self-sufficiency of world regions coping with potentially extreme events affecting large territories; for example, droughts may reduce yields, restrict trading, increase prices, and so on.

The combination of robust ex-ante and ex-post solutions is evaluated by stochastic GLOBIOM for 28 world regions accounting for interdependent trade and respecting security constraints. We find that, in many regions, current policies relying on degenerated solutions from deterministic models are not robust. The results of stochastic and deterministic GLOBIOM are compared at the global level showing that while the 
(a) Rice

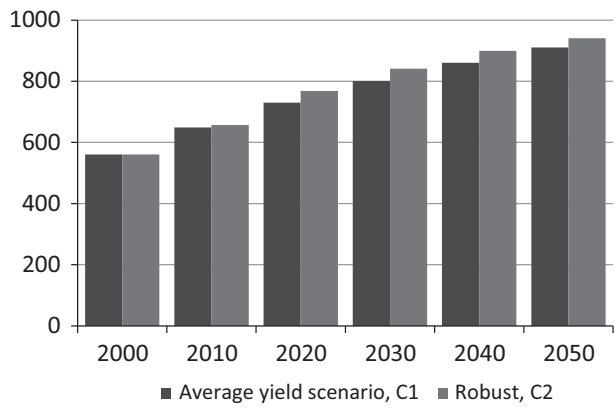

(c) Rape.

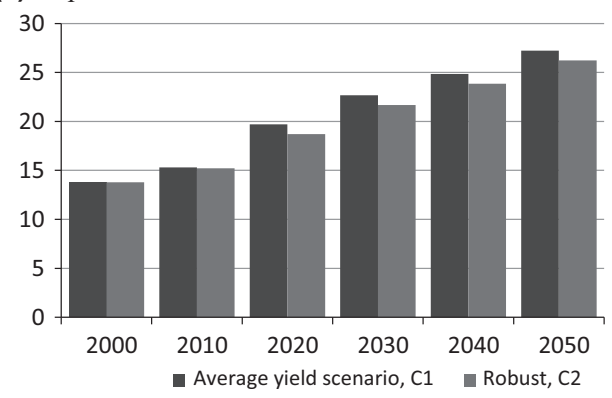

(b) Wheat

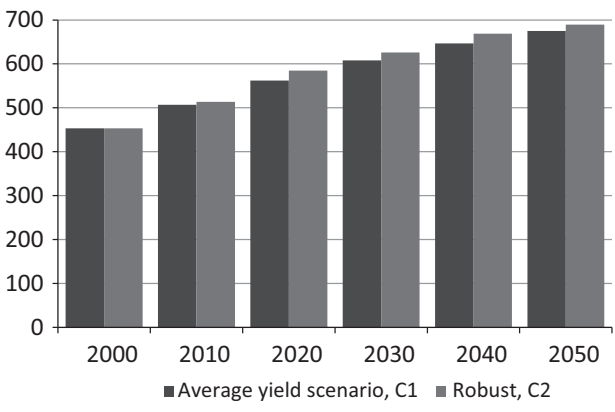

(d) Sunflower.

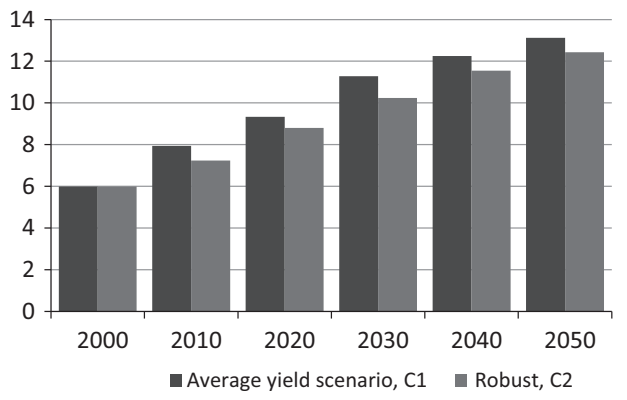

Figure 4. Global demand for selected agricultural commodities calculated using deterministic GLOBIOM under the average yield scenario (Average yield scenario, C1); using stochastic GLOBIOM (Robust, C2). Horizontal axis identifies simulation years and vertical axis shows the demand level in thousand tons. (a) Rice; (b) Wheat; (c) Rape; (d) Sunflower.

robust land allocation among LUS fulfills FEWES targets in the face of all yield shocks, the policy recommendations from deterministic GLOBIOM are scenariodependent. For example, in some scenarios the demand for crop land may exceed the robust requirement (demand) calculated using stochastic GLOBIOM. In section 4, the benefits of robust solutions derived with stochastic GLOBIOM over the solutions derived by scenario analysis of its deterministic counterpart are measured with the VSS. The calculated VSS indicates the importance of including uncertainties when designing robust solutions.

\section{Supporting Information}

Additional Supporting Information may be found in the online version of this article: Appendix S1. Detailed description of stochastic GLOBIOM.

\section{References}

Agarwal, C., Green, G., Grove, J., Evans, T. and Schweik, C. A Review and Assessment of Land-Use Change Models: Dynamics of Space, Time, and Human Choice, USDA Forest Service General Report No. NE-297, 2002.

Arrow, K. J. and Fisher, A. C. 'Preservation, uncertainty and irreversibility', Quarterly Journal of Economics, Vol. 88, (1974) pp. 312-319.

Beach, R., Zhen, C., Rejesus, R., Sinha, P., Lentz, A., Vedenov, D. and McCarl, B. Climate Change Impacts On Crop Insurance, RTI-USDA Risk Management Agency Report Number No. 0211911, 2010. 
Birge, J. R. 'The value of the stochastic solution in stochastic linear programs with fixed recourse', Mathematical Programming, Vol. 24, (1982) pp. 314-325.

Birge, J. R. and Louveaux, F. Introduction to Stochastic Programming (New York, NY: Springer Verlag, 1997).

Colin, C. and Kunreuther, H. 'Making Decisions about Liability and Insurance: Editors Comments', Journal of Risk and Uncertainty, Vol. 7, (1993) pp. 5-15.

Coyle, W. 'The future of biofuels: A global perspective', Amber Waves (ERS Publication), Vol. 5, (2007) pp. 24-29.

Deaton, A. and Laroque, G. 'Competitive storage and commodity price dynamics', Journal of Political Economy, Vol. 104, (1996) pp. 896-923.

Ermoliev, Y. and Hordijk, L. 'Global changes: Facets of robust decisions', in: K. Marti, Y. Ermoliev, M. Makowski and G. Pflug (eds.), Coping with Uncertainty: Modeling and Policy Issue (Berlin, Germany: Springer Verlag, 2003).

Ermoliev, Y. and Jastremski, A. Stochastic Models in Economics (Moscow, Russia: Nauka, 1979).

Ermoliev, Y. and Norkin, V. 'On nonsmooth and discontinuous problems of stochastic systems optimization', European Journal of Operational Research, Vol. 101, (1997) pp. 230-244.

Ermoliev, Y. and von Winterfeldt, D. 'Systemic risk and security management', in: Y. Ermoliev, M. Makowski and K. Marti (eds.), Managing Safety of Heterogeneous Systems: Lecture Notes in Economics and Mathematical Systems (Berlin, Heidelberg, Germany: Springer Verlag, 2012, pp. 19-49.

Ermoliev, Y. and Wets, R. J.-B. Numerical Techniques for Stochastic Optimization (Heidelberg, Germany: Springer Verlag, 1988).

Ermoliev, Y., Ermolieva, T., MacDonald, G. and Norkin, V. 'Stochastic optimization of insurance portfolios for managing exposure to catastrophic risks', Annals of Operations Research, Vol. 99, (2000a) pp. 207-225.

Ermoliev, Y., Ermolieva, T., MacDonald, G. and Norkin, V. 'Insurability of catastrophic risks: The stochastic optimization model', Optimization, Vol. 47, (2000b) pp. 251-265.

FAO. The State of Food Insecurity in the World, Report of Food and Agriculture Organization of the United Nations, Rome, 2009.

FAO. The State of the World's Land and Water Resources for Food and Agriculture (SOLAW) - Managing Systems at Risk, Report of Food and Agriculture Organization of the United Nations, Rome, 2011.

FAO, IFAD, IMF, OECD, UNCTAD, WFP, the World Bank, the WTO, IFPRI, UNHLTF. Price Volatility in Food and Agricultural Markets: Policy Responses, Policy Report, 2011. Available at: http://www.oecd.org/tad/agricultural-trade/48152638.pdf (last accessed 20.08.2015).

GFDRR. Managing Disaster Risks for a Resilient Future: A Work Plan for the Global Facility for Disaster Reduction and Recovery, 2016-2018 (Washington, DC: World Bank Global Facility for Disaster Reduction and Recovery (GFDRR), 2015).

Gielen, D. J., Gerlagh, T. and Bos, A. J. M. MATTER 1.0 - A MARKAL Energy and Materials System - Model Characterisation, ECN Report No. ECN-C-98-085 (ECN, Petten, the Netherlands, 1998).

Gielen, D. J., Bos, A. J. M., deFeber, M. A. P. C. and Gerlagh, T. Biomass for Greenhouse Gas Emission Reduction Task 8: Optimal Emission Reduction Strategies for Western Europe, ECN Report No. ECN-C-00-001 (ECN, Petten, the Netherlands, 2000).

Grain. Against the Grain: Land grabbing for biofuels must stop, Grain Report, 2013. Available at: http://www.grain.org/article/entries/4653-land-grabbing-for-biofuels-must-stop (last accessed 05.04.2015).

Gustafson, R. L. 'Implications of recent research on optimal storage rules', Journal of Farm Economics, Vol. 38, (1958) pp. 290-300.

Havlík, P., Schneider, U. A., Schmid, E., Boettcher, H., Fritz, S., Skalský, R., Aoki, K., de Cara, S., Kindermann, G., Kraxner, F., Leduc, S., McCallum, I., Mosnier, A., Sauer, T. and Obersteiner, M. 'Global land-use implications of first and second generation biofuel targets', Energy Policy, Vol. 39, (2011) pp. 5690-5702. 
Headey, D. Rethinking the Global Food Crisis: The Role of Trade Shocks, Discussion Paper No. 00958 (IFPRI, 2010). Available at: http://www.ifpri.org/sites/default/files/publications/ ifpridp00958.pdf (last accessed 05.04.2015).

James, W. P. T. and Schofield, E. C. Human Energy Requirements: A Manual for Planners and Nutritionists (Oxford, UK: Oxford Medical Publications under arrangement with FAO, 1990).

Kall, P. and Mayer, J. Stochastic Linear Programming: Models, Theory, and Computation (New York, NY: Springer-Verlag, 2004).

Kok, K. and Winograd, M. 'Modeling land-use change for Central America, with reference to the impact of Hurricane Mitch', Ecological Modelling, Vol. 149, (2002) pp. 53-69.

Leclère, D., Havlík, P., Fuss, S., Schmid, E., Mosnier, A., Walsh, B., Valin, H., Herrero, M., Khabarov, N. and Obersteiner, M. 'Climate change induced transformations of agricultural systems: Insights from a global model', Environmental Research Letters, Vol. 9, (2014) pp. 124018.

Liu, J., Williams, J. R., Zehnder, A. J. and Yang, H. 'GEPIC - modelling wheat yield and crop water productivity with high resolution on a global scale', Agricultural Systems, Vol. 94, (2007) pp. 478-493.

Markowitz, H. M. 'Portfolio selection', The Journal of Finance, Vol. 7, (1952) pp. 77-91.

Marti, K. Stochastic optimization methods (Heidelberg, Germany: Springer Verlag, 2008).

McCarl, B. A. and Schneider, U. 'U.S. agriculture's role in a greenhouse gas mitigation world: An economic perspective', Review of Agricultural Economics, Vol. 22, (2000) pp. 134-159.

Myers, N. 'Environment and security', Foreign Policy, Vol. 74, (1989) pp. 23-41.

OECD. Emerging Risks in the $21^{\text {st }}$ Century: An Agenda for Action (Paris, France: OECD Publication Service, 2003).

Prekopa, A. 'Numerical solution of probabilistic constrained programming problems', in Y. Ermoliev and R. J.-B. Wets (eds.), Numerical Techniques for Stochastic Optimization (New York, NY: Springer Verlag, 1988, pp. 123-139).

Swiss Re. Partnering for Food Security In Emerging Markets (Einsiedeln, Germany: Sigma, EA Druck Verlag AG, 2013).

Rockafellar, R. T. and Uryasev, S. 'Optimization of conditional value-at-risk', Journal of Risk, Vol. 2, (2000) pp. 21-41.

Spinoni, J., Naumann, G., Vogt, J. and Barbosa, P. 'The biggest drought events in Europe from 1950 to 2012', Journal of Hydrology: Regional Studies, Vol. 3, (2015) pp. 509-524.

Stiglitz, J. E. 'Incentives and risk sharing in sharecropping', Review of Economic Studies, Vol. 41, (1974) pp. 219-255.

US Department of Energy. World Biofuels Production Potential: Understanding the Challenges To Meeting the U.S. Renewable Fuel Standard (Washington, DC: Office of Policy and International Affairs, 2008).

Valin, H., Havlik, P., Mosnier, A., Herrero, M., Schmid, E. and Obersteiner, M. 'Agricultural productivity and greenhouse gas emissions: Trade-offs or synergies between mitigation and food security?', Environmental Research Letters, Vol. 8, (2013) pp. 035019.

Veldkamp, A. and Fresco, L. 'CLUE-CR: An integrated multi-scale model to simulate land use change scenarios in Costa Rica', Ecological Modelling, Vol. 91, (1996) pp. 231-248.

Verburg, P., Chen, Y. and Veldkamp, A. 'Spatial explorations of land-use change and grain production in China', Agriculture, Ecosystems and Environment, Vol. 82, (2000) pp. 333-354.

WHO. Energy and Protein Requirements, Report of a joint FAO/WHO/UNU expert consultation, WHO Technical Report Series No. 724, 1985. Available at: http://www.fao.org/ doCReP/003/aa040e/AA040E00.htm (last accessed 30.05.2015).

Wright, B. 'The economics of grain price volatility', Applied Economic Perspectives and Policy, Vol. 33, (2011) pp. 32-58.

Zou, X., Zhai, P. and Zhang, Q. 'Variations in droughts over China: 1951-2003', Geographical Research Letters, Vol. 32, (2005) p. L04707. 